\title{
GPS/INS/Seeker Integrated Navigation System for the Case of GPS Blockages
}

Woo Hyun Kim, Jang Gyu Lee

School of Electrical Engineering and Computer Science, Seoul National University,

133-dong, ASRI \#610, San 56-1, Sillim-dong, Gwanak-gu, Seoul, 151-742, South Korea

Hyung Keun Lee

School of Electronics, Telecommunication \& Computer Eng., Korea Aerospace University, 200-1, Hwajon-dong, Deokyang_gu, Goyang-city, Kyunggi-do, 412-791, South Korea

Chan Gook Park

School of Mechanical and Aerospace Engineering, Seoul National University,

San 56-1, Sillim-dong, Gwanak-gu, Seoul, 151-744, South Korea

Abstract. When GPS blockage occurs for a loosely coupled GPS/INS system, its navigation error diverges. To deal with such cases, this paper introduces an integration scheme for GPS, INS, and an image sensor. The proposed integration scheme is attractive in that it accomplishes the position and velocity accuracy improvement by the angular information only. The angular information is provided by the gimbal angles of the image sensor. A realistic scenario is studied by a simulation to demonstrate that the GPS/INS/Image integrated navigation system works effectively.

Keywords. Image sensor, inertial navigation system (INS), GPS/INS/Image Integrated Navigation System, GPS/INS loosely coupled system

\section{Introduction}

Loosely coupled GPS/INS is one of the most popular integration schemes for modern navigation systems. This scheme performs well when GPS signal conditions are good. However when a GPS signal blockage occurs, its navigation error diverges considerably even within a few minutes(P.S. Maybeck, 1994). GPS blockages occur due to physical obstructions, vehicle dynamics, signal jamming, receiver faults, and satellite faults. Against these cases, additional information is required to compensate the GPS blockage error(Titterton D.H., 1997). For such cases, an image sensor might be a good source of aiding information to prevent the navigation error divergence. In Fig. 1, a conventional configuration for a gimballed image sensor is illustrated. As shown in Fig. 1, the image sensor is suspended by a two-axis gimbal system (Ryu Moo Young, 2005). Thus, the pitch and yaw angle measurements regarding the line-of-sight (LOS) information can be provided by the image sensor.

For the integration of an INS and an image sensor, a previous study focused on the INS attitude alignment by utilizing a star tracker (Vathal S., 1987). Extending the previous research work, this paper investigates the performance improvement of INS by an image sensor in the cases of GPS blockages. As compared with the previous research work that mainly deal with the attitude accuracy improvement, this paper focuses on the position and velocity accuracy improvement.

This paper is organized as follows. At first, important coordinate systems are introduced. Next, measurement equations are derived. Thirdly, a simulation result is demonstrated to validate the performance of the GPS/INS/Image integration scheme. Finally, concluding remarks will be given.

Fig. 1 Configuration of a two-axis gimbaled image sensor. (Ryu Moo Young, 2005)

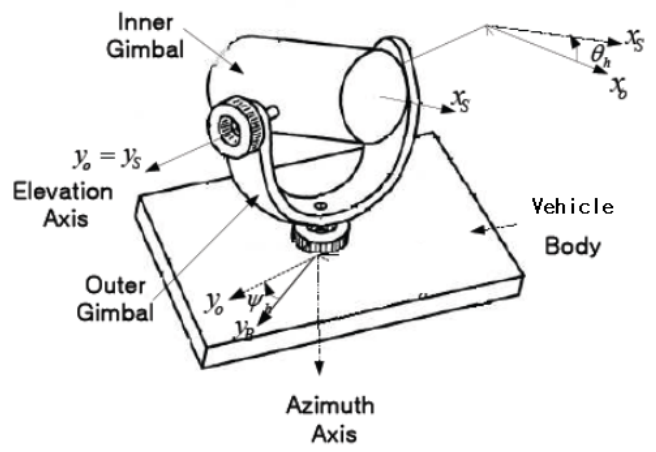

\section{Design of GPS/INS/Image integrated navigation system}

When an image sensor searches and locks on to a landmark's image at the center of the image plane, at least 4 frames are necessary to account for this 
geometry. They are the navigation frame (n-frame), the body frame (b-frame), the image sensor frame (s-frame), and the landmark frame (l-frame) as shown in Fig. 2. The transformation matrix $C_{s}^{n}$ from the image sensor frame to the navigation frame is decomposed into a transformation matrix $C_{s}^{b}$ from the image sensor frame to the body frame and the transformation matrix $C_{b}^{n}$ from the body frame to the navigation frame.

$$
C_{s}^{n}=C_{b}^{n} C_{s}^{b}
$$

As shown in Fig. 2, the unit LOS vector $e$ coincides with the x-direction basis vector $X_{s}$ of the image sensor frame. Eq. (2) expresses the LOS vector $e$ in the image sensor frame.

$$
e^{s}=\left[\begin{array}{lll}
1 & 0 & 0
\end{array}\right]^{T}
$$

Eq. (3) shows how the LOS vector $e$ with respect to the navigation frame can be obtained by utilizing the transformation matrix $C_{s}^{n}$.

$$
e^{n}=C_{s}^{n} e^{s}
$$

The exact LOS vectors shown in Eq. (3) cannot be obtained in practice. Instead, an estimate of it can be obtained as follows.

$$
\widehat{C}_{s}^{n}=\hat{C}_{b}^{n} \widetilde{C}_{s}^{b}
$$

where

$$
\begin{aligned}
\hat{C}_{b}^{n} & =C_{b}^{n}(\mathrm{I}-\langle\Phi\rangle) \\
\widetilde{C}_{s}^{b} & =C_{s}^{b}(\mathrm{I}-\mathrm{E})
\end{aligned}
$$

$\hat{C}_{s}^{n}$ : estimated transformation matrix from the image sensor frame to the navigation frame

$\hat{C}_{b}^{n}$ : transformation matrix from the body frame to the navigation frame estimated by the INS $\widetilde{C}_{s}^{b}$ : transformation matrix from the image sensor frame to the body frame measured by the gimbal angles.

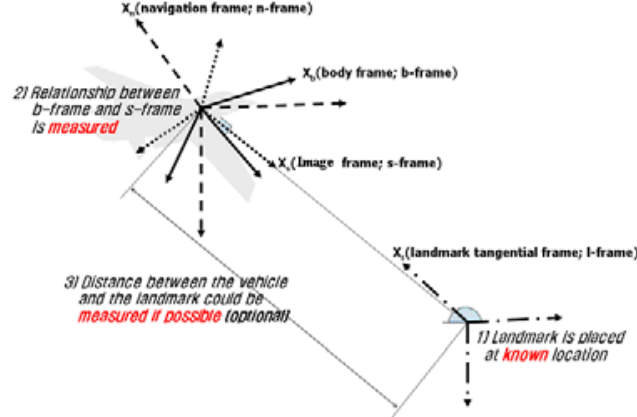

Fig. 2 Geometry of coordinate systems

$\Phi$ : skew symmetric matrix of the INS attitude error $\mathrm{E}$ : skew symmetric matrix of the gimbal measurement error of the image sensor

The matrix $C_{s}^{b}$ in Eq. (5) is defined as follows

$C_{s}^{b}=\left[\begin{array}{ccc}\cos \psi_{s} \cos \theta_{s} & -\sin \psi_{s} & \cos \psi_{s} \sin \theta_{s} \\ \sin \psi_{s} \cos \theta_{s} & \cos \psi_{s} & \sin \psi_{s} \sin \theta_{s} \\ -\sin \theta_{s} & 0 & \cos \theta_{s}\end{array}\right]$

To construct an indirect Kalman filter, an indirect measurement should be formed as the difference between the raw measurement and the filter's estimate of its equivalency as follows.

$$
z=\hat{y}_{\text {INS }}-\tilde{y}_{\text {meas. }}
$$

$\hat{y}_{I N S}:$ value estimate by INS

$\tilde{y}_{\text {meas. }}:$ measured value

The gimbal angles provided by the image sensor indicate the difference between the body frame and the image sensor frame. Thus, they do not indicate the angles between the body frame and the navigation frame. Due to this characteristic, it was determined that the straightforward derivation like Eq. (6) is difficult and too complex. To simplify this problem, the unit LOS vector $e^{n}$ from the vehicle body to the landmark is utilized as follows.

1) Derive $\hat{e}_{\text {INS }}^{n}$.

2) Derive $\hat{e}_{\text {landmark }}^{n}$.

3) Subtract $\hat{e}_{\text {INS }}^{n}$ from $\hat{e}_{\text {landmark }}^{n}$

$\hat{e}_{\text {INS }}^{n}:$ unit LOS vector formed by the image sensor gimbal angle measurements and INS's attitude information.

$\hat{e}_{\text {landmark }}^{n}$ : unit LOS vector formed by position of landmark and INS's position information 
Indirect measurement is formed as the difference between $\hat{e}_{I N S}^{n}$ and $\hat{e}_{\text {landmark }}^{n}$. As a result, the indirect measurement is obtained as follows

$$
\begin{aligned}
& Z_{\mathrm{LOS}}=\hat{e}_{\text {landmark }}^{n}-\hat{e}_{\text {INS }}^{n} \\
& =\delta e_{\text {landmark }}^{n}-\delta e_{\text {INS }}^{n} \\
& =f(\delta L, \delta l, \delta h)-g(\Phi, \mathrm{E}) \\
& Z_{\mathrm{GPS}}=\left[\begin{array}{l:l:l:l:l:l}
I_{3 \times 3} & 0_{3 \times 3} & 0_{3 \times 3} & 0_{3 \times 3} & 0_{3 \times 3} & 0_{3 \times 2}
\end{array}\right] \\
& Z_{\text {GPS-INS-LOS }}=\left[\begin{array}{c}
Z_{\mathrm{GPS}} \\
Z_{\mathrm{LOS}}
\end{array}\right]
\end{aligned}
$$

As shown above, $\delta e_{\text {landmark }}^{n}$ is affected by the position error, $\delta e_{I N S}^{n}$ is affected by the function of vehicle's attitude error and the gimbal measurement error. For convenience, they are denoted by $f(\delta L, \delta l, \delta h)$ and $g(\Phi, \mathrm{E})$, respectively.

\subsection{Derivation of $\hat{e}_{I N S}^{n}$}

To estimate the LOS vector resolved with respect to the navigation frame, we utilize Eq. (4). For this purpose, the attitude information of the INS and the gimbal angle measurements of the image sensor are utilized. The LOS vector apparently coincides with the $\mathrm{x}$-axis basis vector of the LOS frame. Thus, the LOS vector resolved with respect to the navigation frame can be computed as follows.

$$
\hat{e}_{I N S}^{n}=\hat{C}_{s}^{n}\left[\begin{array}{lll}
1 & 0 & 0
\end{array}\right]^{T}
$$

The transformation matrix $\hat{C}_{s}^{n}$ shown in Eq. (8) contains error terms due to Eqs. (5a) and (5b). It is summarized as follows.

$$
\begin{aligned}
\hat{C}_{s}^{n}=C_{s}^{n}+\delta & \delta C_{s}^{n} \\
\delta C_{s}^{n} & =\hat{C}_{s}^{n}-C_{s}^{n} \\
& =-\hat{C}_{s}^{n} \mathrm{E}-\hat{C}_{b}^{n}\langle\Phi\rangle \widetilde{C}_{s}^{b}
\end{aligned}
$$

Combining Eqs. (3), (8), and (9), $\delta e_{I N S}^{n}$ can be derived as follows.

$$
\begin{aligned}
& \hat{e}_{I N S}^{n}=e_{I N S}^{n}+\delta e_{I N S}^{n} \\
& \delta e_{I N S}^{n}=\delta C_{s}^{n}\left[\begin{array}{lll}
1 & 0 & 0
\end{array}\right]^{T}=g(\Phi, \mathrm{E}) \\
& =-\left[\begin{array}{c}
\delta \gamma\left[C_{1}\right]+\delta \alpha\left[A_{1}\right]-\delta \beta\left[B_{1}\right]+\phi_{z}\left[P Z_{1}\right]-\phi_{y}\left[P Y_{1}\right] \\
\hdashline \gamma\left[C_{2}\right]+\delta \alpha\left[A_{2}\right]-\delta \beta\left[B_{2}\right]+\phi_{z}\left[P Z_{2}\right]-\phi_{y}\left[P Y_{2}\right] \\
\hdashline \delta \gamma\left[C_{3}\right]+\delta \alpha\left[A_{3}\right]+\delta \beta\left[B_{3}\right]+\phi_{z}\left[P Z_{3}\right]-\phi_{y}\left[P Y_{3}\right]
\end{array}\right] \\
& =-\left[\begin{array}{c}
(1,1) \\
\frac{(2,1)}{(3,1)}
\end{array}\right]
\end{aligned}
$$

As shown in Eq. (10), $\delta e_{I N S}^{n}$ is a 3 by 1 matrix. Denoting $\delta \alpha, \delta \beta, \delta \gamma, \phi_{y}, \phi_{z}$ as the roll error, pitch error, yaw error, gimbal pitch angle error, and gimbal yaw angle error, respectively, the elements of $\delta e_{I N S}^{n}$ are summarized as follows.

$$
\begin{aligned}
& {\left[\int \delta \gamma\left[\begin{array}{l}
{\left[\sin \phi_{b} \sin \theta_{b} \cos \psi_{b}-\cos \phi_{b} \sin \psi_{b}\right]\left[\cos \theta_{s} \cos \psi_{s}\right]} \\
-\left[\cos \theta_{b} \cos \psi_{b}\right]\left[\cos \theta_{s} \sin \psi_{s}\right]
\end{array}\right]\right.} \\
& +\delta \alpha\left[\begin{array}{l}
{\left[\cos \phi_{b} \sin \theta_{b} \cos \psi_{b}+\sin \phi_{b} \sin \psi_{b}\right]\left[\cos \theta_{s} \sin \psi_{s}\right]} \\
+\left[\sin \phi_{b} \sin \theta_{b} \cos \psi_{b}-\cos \phi_{b} \sin \psi_{b}\right]\left[\sin \theta_{s}\right]
\end{array}\right] \\
& -\delta \beta\left[\begin{array}{l}
{\left[\cos \theta_{b} \cos \psi_{b}\right]\left[\sin \theta_{s}\right]} \\
+\left[\cos \phi_{b} \sin \theta_{b} \cos \psi_{b}\right.
\end{array}\right. \\
& (1,1)=\left(\begin{array}{l}
\left(\begin{array}{l}
{\left[\cos \theta_{b} \cos \psi_{b}\right]\left[-\sin \psi_{s}\right]} \\
+\left[\sin \phi_{b} \sin \theta_{b} \cos \psi_{b}-\cos \phi_{b} \sin \psi_{b}\right] \\
{\left[\cos \psi_{s}\right]}
\end{array}\right)\left[\phi_{z}\right] \\
+\left(\begin{array}{l}
{\left[\cos \theta_{b} \cos \psi_{b}\right]\left[\sin \theta_{s} \cos \psi_{s}\right]} \\
+\left[\sin \phi_{b} \sin \theta_{b} \cos \psi_{b}-\cos \phi_{b} \sin \psi_{b}\right] \\
{\left[\sin \theta_{s} \sin \psi_{s}\right]} \\
+\left[\cos \phi_{b} \sin \theta_{b} \cos \psi_{b}+\sin \phi_{b} \sin \psi_{b}\right] \\
{\left[\cos \theta_{s}\right]}
\end{array}\right)\left[\phi_{y}\right]
\end{array}\right)
\end{aligned}
$$

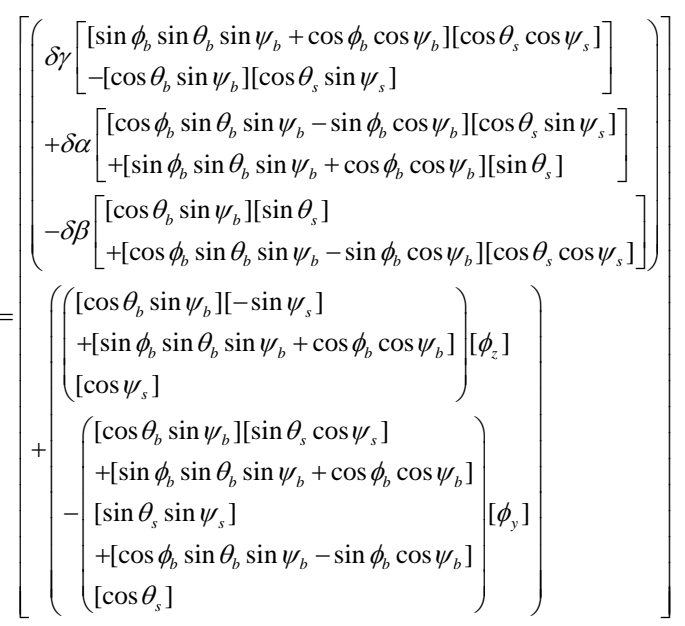




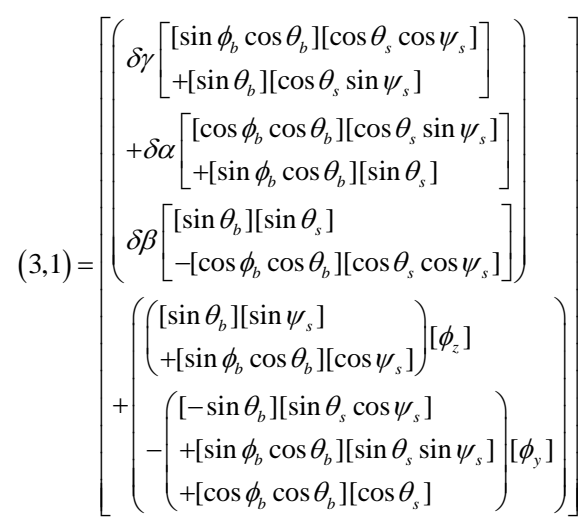

\subsection{Derivation of $\hat{e}_{\text {landmark }}^{n}$}

If exact positions of the landmark and the vehicle are known, the unit LOS vector $e^{n}$ can be obtained, differently from (3), as follows.

$$
\begin{gathered}
e^{n}=D^{n}\left(\left\|D^{n}\right\|\right)^{-1} \\
D^{n}=C_{L}^{n} \Delta L
\end{gathered}
$$

where Eq. (12) denotes the position difference between the landmark and the vehicle. As shown in Eq. (12), it is the function of differences in latitude, longitude, and height.

$$
\Delta L=\left[\begin{array}{lll}
L & l & h
\end{array}\right]_{\text {landmark }}^{T}-\left[\begin{array}{lll}
L & l & h
\end{array}\right]_{\text {vehicle }}^{T}
$$

The scaling matrix $C_{L}^{n}$ shown in Eq. (12) is purposed to transform the latitude, longitude, and height differences to the difference vector resolved with respect to the locally-level navigation frame.

$$
C_{L}^{n}=\left[\begin{array}{c:c:c}
R_{m}+h & 0 & 0 \\
\hdashline 0 & \left(R_{t}+h\right) \cos L & 0 \\
\hdashline 0 & 0 & -1
\end{array}\right]
$$

In Eq. (14), $R_{m}$ denotes the meridian radius of curvature and $R_{t}$ denotes the transverse radius of curvature of the earth.

Since it is hard to obtain the exact position of vehicle and the exact position of vehicle is an object of estimation, it is not available in practice. Instead, the estimated latitude, longitude, and height information of the INS is utilized as follows.

$$
\begin{aligned}
& \hat{e}_{\text {landmark }}^{n}=\widehat{D}^{n}\left(\left\|\widehat{D}^{n}\right\|\right)^{-1} \\
& \hat{D}^{n}=C_{L}^{n} \Delta \hat{L} \\
& \Delta \hat{L}=\left[\begin{array}{lll}
L & l & h
\end{array}\right]_{\text {landmark }}^{T}-\left[\begin{array}{lll}
\hat{L} & \hat{l} & \hat{h}
\end{array}\right]_{\text {INS }}^{T}
\end{aligned}
$$

Combining Eqs. (16) and (17), the following relationships can be derived.

$$
\begin{aligned}
& \widehat{D}^{n}=C_{L}^{n} \Delta L-C_{L}^{n}\left[\begin{array}{lll}
\delta L & \delta l & \delta h
\end{array}\right]_{I N S}^{T} \\
& \hat{D}^{n}=D^{n}+\delta D^{n} \\
& \delta D^{n}=-C_{L}^{n}\left[\begin{array}{lll}
\delta L & \delta l & \delta h
\end{array}\right]_{I N S}^{T} \\
& {\left[\begin{array}{lll}
\delta L & \delta l & \delta h
\end{array}\right]_{I N S}^{T}=\left[\begin{array}{lll}
\hat{L} & \hat{l} & \hat{h}
\end{array}\right]_{I N S}^{T}-\left[\begin{array}{lll}
L & l & h
\end{array}\right]_{\text {vehicle }}^{T}}
\end{aligned}
$$

The estimated LOS vector $\hat{e}_{\text {landmark }}^{n}$ can be obtained by applying the perturbation method to Eqs. (11) and (15) with having such error sources.

$$
\begin{aligned}
& \hat{e}_{\text {landmark }}^{n}=e_{\text {landmark }}^{n}+\delta e_{\text {landmark }}^{n} \\
& \hat{e}_{\text {landmark }}^{n}=\frac{\hat{D}^{n}}{\left\|\hat{D}^{n}\right\|}=\frac{D^{n}}{\left\|D^{n}\right\|}+\frac{\partial}{\partial \hat{D}^{n}}\left(\frac{\widehat{D}^{n}}{\left\|\widehat{D}^{n}\right\|} \|_{D^{n}} \delta D^{n}+\right.\text { H.O.T. } \\
& \delta e_{\text {landmark }}^{n}=\frac{\partial}{\partial \hat{D}^{n}}\left(\left.\frac{\widehat{D}^{n}}{\left\|\hat{D}^{n}\right\|}\right|_{D^{n}} \delta D^{n}\right. \\
& \frac{\partial}{\partial \widehat{D}^{n}}\left(\frac{\widehat{D}^{n}}{\left\|\widehat{D}^{n}\right\|}\right)=\mathrm{I}_{3 \times 3} \frac{1}{\left\|\widehat{D}^{n}\right\|}-\frac{\widehat{D}^{n} \widehat{D}^{n^{T}}}{\left\|\widehat{D}^{n}\right\|^{3}} \\
& \delta e_{\text {landmark }}^{n} \approx\left(\mathrm{I}_{3 \times 3}\left(\left\|\widehat{D}^{n}\right\|\right)^{-1}-\widehat{D}^{n} \widehat{D}^{n^{T}}\left(\left\|\hat{D}^{n}\right\|^{3}\right)^{-1}\right) \delta D^{n}=f(\delta L, \delta l, \delta h) \\
& \delta e_{\text {landmark }}^{n}=\text { priori_ } \delta e_{\text {landmark }}^{n} \times\left[\begin{array}{lll}
\delta L & \delta l & \delta h
\end{array}\right]_{\text {INS }}^{T} \\
& =\left[\begin{array}{c:c:c}
(1,1) & (1,2) & (1,3) \\
\hdashline(2,1) & (2,2) & (2,3) \\
\hdashline(3,1) & (3,2) & (3,3)
\end{array}\right]\left[\begin{array}{l}
\delta L \\
\delta l \\
\delta h
\end{array}\right]_{I N S}
\end{aligned}
$$

\subsection{Handling rank deficiency}

As previously explained, the image sensor provides two direct measurements, i.e., pitch and yaw gimbal angles. However, the indirect measurement formed by the direct measurements is 3-by-1 vector. Thus, a rank deficiency can occur, which means that one element is a linear combination of the other two elements. Since the Kalman filter assumes 
independent measurements, the rank deficiency should be eliminated.

To eliminate the rank deficiency, the observation matrix $\mathrm{H}$ should be decomposed into two parts; INS part and image sensor part.

$\left[\begin{array}{lll:lll:lll:lll:lll:ll}(1,1) & (1,2) & (1,3) & 0 & 0 & 0 & A_{1} & -B_{1} & C_{1} & 0 & 0 & 0 & 0 & 0 & 0 & -P Y_{1} & P Z_{1}\end{array}\right]$ $H=$\begin{tabular}{lllllllllll:lll:lll:ll}
$(2,1)$ & $(2,2)$ & $(2,3)$ & 0 & 0 & 0 & $A_{2}$ & $-B_{2}$ & $C_{2}$ & 0 & 0 & 0 & 0 & 0 & 0 & $-P Y_{2}$ & $P Z_{2}$ \\
\hline$(3,1)$ & $(3,2)$ & $(3,3)$ & 0 & 0 & 0 & $A_{2}$ & $B_{3}$ & $C_{3}$ & 0 & 0 & 0 & 0 & 0 & 0 & $-P Y_{3}$ & $P Z_{3}$
\end{tabular}

$\left.\begin{array}{lll:lll:lll:lll:lll:lll}(3,1) & (3,2) & (3,3) & 0 & 0 & 0 & A_{3} & B_{3} & C_{3} & 0 & 0 & 0 & 0 & 0 & 0 & -P Y_{3} & P Z_{3}\end{array}\right]$

$H_{\text {INS }}=\left[\begin{array}{ccc:ccc:ccc:ccc:ccc}(1,1) & (1,2) & (1,3) & 0 & 0 & 0 & A_{1} & -B_{1} & C_{1} & 0 & 0 & 0 & 0 & 0 & 0 \\ \hdashline(2,1) & (2,2) & (2,3) & 0 & 0 & 0 & A_{2} & -B_{2} & C_{2} & 0 & 0 & 0 & 0 & 0 & 0 \\ \hdashline(3,1) & (3,2) & (3,3) & 0 & 0 & 0 & A_{3} & B_{3} & C_{3} & 0 & 0 & 0 & 0 & 0 & 0\end{array}\right]$

$$
H_{\mathrm{LOS}}=\left[\begin{array}{cc}
-P Y_{1} & P Z_{1} \\
\hdashline-P Y_{2} & P Z_{2} \\
\hdashline-P Y_{3} & P Z_{3}
\end{array}\right]
$$

Meanwhile, by the singular value decomposition, the image sensor part observation matrix can be rewritten as follows.

$$
\begin{aligned}
& H_{\mathrm{LOS}}=U S V^{T} \\
& U U^{T}=I \\
& V V^{T}=I
\end{aligned}
$$

Where $U$ and $V$ denote orthonormal matrix and $S$ denotes quasi-diagonal matrix with the singular value as its diagonal elements, respectively. If we denote $B$ as the first two columns of the orthonormal matrix $U$, it can be verified that $B^{T} H$ is a full rank matrix and the following relationships are satisfied

$$
\begin{aligned}
\breve{Z}_{\mathrm{LOS}} & =B^{T} Z_{\mathrm{LOS}} \\
\breve{H}= & B^{T} H \\
\breve{H}_{\mathrm{LOS}} & =B^{T} H_{\mathrm{LOS}} \\
\widehat{Z}_{\mathrm{LOS}} & =\breve{H}_{\mathrm{LOS}}^{-1} \breve{Z}_{\mathrm{LOS}} \\
& =\left(B^{T} H_{\mathrm{LOS}}\right)^{-1} B^{T} Z_{\mathrm{LOS}}
\end{aligned}
$$

It can be verified that $\hat{Z}_{\text {Los }}$ shown in Eq. (24) is a 2by- 1 vector and is free of rank deficiency. As a result, the Kalman filter utilizes the following full rank measurement vector instead of that shown in Eq. (7).

$$
Z_{\text {GPS-INS-LOS }}=\left[\begin{array}{l}
Z_{G P S} \\
Z_{\text {LOS }}
\end{array}\right]
$$

\section{Simulation and result}

To assess the performance of the GPS/INS/image integrated navigation system, a simulation has been performed. For the simulation, the initial latitude error, longitude error, and height error are set as 40/Ro[rad], 40/Ro[rad], and 40[m], respectively, where Ro denotes the mean radius of the earth. The initial velocity error in each direction is set as $10[\mathrm{~m} / \mathrm{sec}]$, respectively. The initial roll error of vehicle, pitch error of vehicle, and yaw error of vehicle are set as 1[deg], 1[deg], and 5[deg], respectively. The scale factor, misalignment error, white noise, and random constant of the accelerometer are set as 200[ppm], 10[arcsec], 5[ $\mu \mathrm{g}]$, and 100[ $\mu \mathrm{g}$ ], respectively.

It is assumed that GPS blockage occurs at from 121 [sec] to 180 [sec] during the flight. To enhance the observability, an S-shape turn trajectory is applied to vehicle trajectory. The state variables used for the loosely-coupled GPS/INS system are the position errors, velocity errors, attitude errors, accelerometer bias, and gyro drift (Young Bum Park, 2001). The proposed GPS/INS/Image integrated navigation system augments the conventional 15 error states by the two bias values in the image sensor's pitch and yaw measurements.

$$
\begin{aligned}
& F_{\text {GPS-INS-LOS }}=\left[\begin{array}{c:c:c:c:c:c}
F_{11} & F_{12} & 0_{3 \times 3} & 0_{3 \times 3} & 0_{3 \times 3} & 0_{3 \times 2} \\
\hdashline F_{21} & F_{22} & F_{23} & F_{24} & 0_{3 \times 3} & 0_{3 \times 2} \\
\hdashline F_{31} & F_{32} & F_{33} & 0_{3 \times 3} & F_{35} & 0_{3 \times 2} \\
\hdashline 0_{3 \times 3} & 0_{3 \times 3} & 0_{3 \times 3} & 0_{3 \times 3} & 0_{3 \times 3} & 0_{3 \times 2} \\
\hdashline 0_{3 \times 3} & 0_{3 \times 3} & 0_{3 \times 3} & 0_{3 \times 3} & 0_{3 \times 3} & 0_{3 \times 2} \\
\hdashline 0_{2 \times 3} & 0_{2 \times 3} & 0_{2 \times 3} & 0_{2 \times 3} & 0_{2 \times 3} & F_{66}
\end{array}\right] \\
& \delta X=\left[\begin{array}{l:l}
\delta X_{\text {INS }}^{T} & \delta X_{\mathrm{LOS}}^{\mathrm{T}}
\end{array}\right]^{T} \\
& \delta X_{\text {INS }}= \\
& {\left[\begin{array}{lll:lll:lll:lll:lll}
\delta L_{I N S} & \delta l_{I N S} & \delta h_{I N S} & \delta V_{N} & \delta V_{E} & \delta V_{D} & \delta \alpha & \delta \beta & \delta \gamma & \nabla_{X} & \nabla_{Y} & \nabla_{Z} & \varepsilon_{X} & \varepsilon_{Y} & \varepsilon_{Z}
\end{array}\right]^{T}} \\
& \delta X_{\mathrm{LOS}}=\left[\begin{array}{ll}
\phi_{y} & \phi_{z}
\end{array}\right]^{T}
\end{aligned}
$$

Fig. 3, Fig. 4, and Fig. 5 show position, velocity, and attitude errors of the two integration schemes, respectively. As shown in all figures, the GPS/INS loosely coupled system shows an accumulation of navigation error during the GPS blockage between 121 and 180 second. As compared, the proposed GPS/INS/Image integrated navigation system generates a trajectory which is close to the truth trajectory during all the time. Thus, it can be concluded that it is possible to bound all the navigation errors by utilizing only the two angle 
measurements. It is interpreted that the error bounding is possible though the direct range information between the vehicle and the landmark is not available. The attractive error bounding feature is interpreted due to the observability enhancement brought by the vehicle's S-turn.

Fig. 3 Comparison position errors.

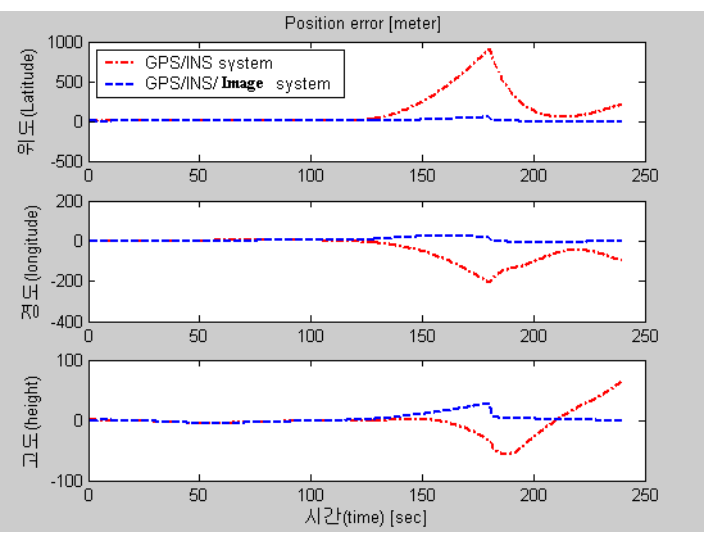

Fig. 4 Comparison velocity errors.

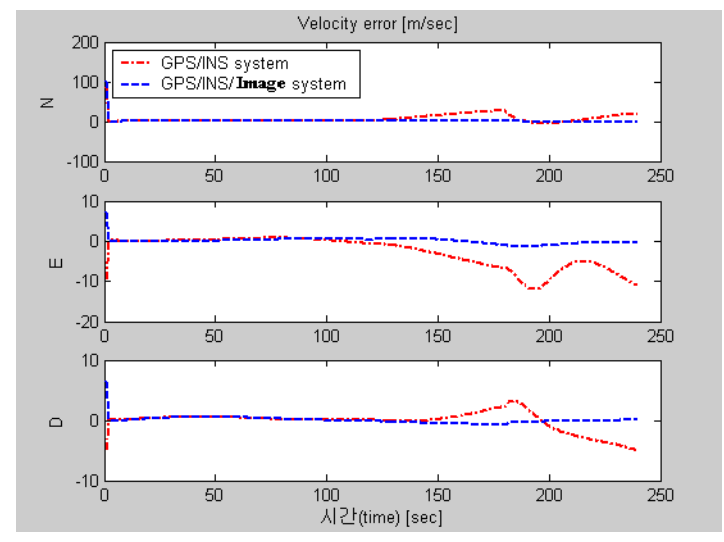

Fig. 5 Comparison attitude errors.

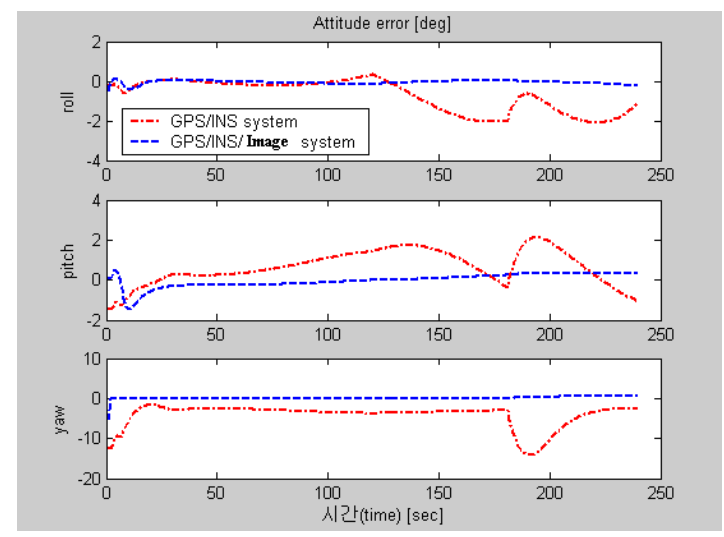

\section{Conclusion}

The paper proposed a new GPS/INS/image integration scheme to prevent the INS error accumulation during GPS blockages. For the purpose, two gimbal measurements of the image sensor are utilized. Assuming that the image sensor acquires a known landmark in the center of its image plane, a simulation was performed. By the simulation result, it was confirmed that it is possible to bound all the navigation errors by utilizing the image sensor's two angle measurements only.

\section{Acknowledgements}

This work has been supported by the Agency for Defense Development (ADD) and the Automatic Control Research Center (ACRC), Seoul National University.

\section{References}

George M. Siouris(2004), Missile Guidance and Control Systems, Springer, pp.102 103.

M. R. Ananthasayanam, A. K. Sarkar, A. Bhattacharya, P. K. Tiwari, and P. Vohra(2005), AIAA Guidance, Navigation, and Control Conference, pp.2 4.

P.S. Maybeck (1994), Stochastic Models, Estimation, and Control, Vol. I, Navtech Book \& Software Store pp.291 297.

Park Young Bum(2001), Design of Integrated INS/GPS/Odometer Navigation System and Its Performance Analysis, Thesis, School of Electronic Engineering and Computer Science, Seoul National University.

Ryu Moo Yong(2005), Feedback Linearization Control of a 2-Axis Gimbal Seeker in BTT Missile, Thesis, School of Electronic Engineering and Computer Science, Seoul National University.

D.H.Titterton, J. L. Weston (1997), Strapdown inertial navigation technology, Peter Peregrinus Ltd., pp.363 394.

S. Vathal (1987), "Spacecraft Attitude Determination Using a Second-Order Nonlinear Filter,"Journal of Guidance and Control, Vol.10, No 6, pp.559 565. 